\title{
Conjunctivitis in COVID-19 patients: frequency and clinical presentation
}

\author{
Noemi Güemes-Villahoz ${ }^{1}$ - Barbara Burgos-Blasco ${ }^{1}$ (D) - Julián García-Feijoó ${ }^{2} \cdot$ Federico Sáenz-Francés $^{2}$. \\ Pedro Arriola-Villalobos ${ }^{2}$. Jose María Martinez-de-la-Casa ${ }^{2}$. Jose Manuel Benítez-del-Castillo ${ }^{2}$. \\ María Herrera de la Muela ${ }^{3}$
}

Received: 1 June 2020 / Revised: 20 July 2020 / Accepted: 25 August 2020 / Published online: 29 August 2020

(C) Springer-Verlag GmbH Germany, part of Springer Nature 2020

\begin{abstract}
Purpose The purpose of this study was to evaluate the frequency and clinical presentation of conjunctivitis in hospitalized patients with COVID-19.

Methods A cross-sectional study was conducted at the Hospital Clinico San Carlos of Madrid, Spain. A total of 301 subjects from the COVID admission unit with laboratory-confirmed SARS-CoV-2 infection were included. The presence and clinical characteristics of conjunctivitis were evaluated. Laboratory, radiological, and clinical results in patients with and without conjunctivitis stratified by sex were analyzed.

Results Of the 301 subjects included, 180 patients (59.8\%) were male and the median age was 72 years (IQ 59-82). Overall, 35 patients $(11.6 \%)$ were diagnosed with acute conjunctivitis. We found no relationship between the COVID-19 severity score and the presence of conjunctivitis $(P=0.17)$. However, conjunctivitis was more frequent in males with moderate clinical severity and in women classified as clinically mild. The natural history of the disease seems to be a rapid self-limited conjunctivitis that improves without treatment and does not affect visual acuity nor associate short-term complications.

Conclusions Approximately, 1 out of 10 hospitalized non-critical COVID-19 patients presents conjunctivitis during the disease. Compared with other viral conjunctivitis, we found distinctive clinical findings that could guide defining and differentiating conjunctivitis in COVID-19 patients.
\end{abstract}

Trial registration number 20/336_E_COVID

Keywords Conjunctivitis $\cdot$ COVID-19 $\cdot$ SARS-CoV-2 $\cdot$ Ocular $\cdot$ Coronavirus

\section{Introduction}

A novel coronavirus $(\mathrm{CoV})$ named severe acute respiratory syndrome coronavirus-2 (SARS-CoV-2) emerged from

Barbara Burgos-Blasco

bburgos171@hotmail.com

1 Department of Ophthalmology, Hospital Clínico San Carlos, Calle del Prof Martín Lagos, s/n, 28040 Madrid, Spain

2 Department of Ophthalmology, Hospital Clinico San Carlos, Instituto de investigación sanitaria del Hospital Clínico San Carlos (IsISSC). IIORC, Universidad Complutense de Madrid, ISCIII (OFTARED), Madrid, Spain

3 Obstetrics and Gynecology Department, Hospital Clinico San Carlos, Instituto de salud de la mujer. Instituto de investigación sanitaria del Hospital Clínico San Carlos (IsISSC), Madrid, Spain
China in December 2019. This virus causes the coronavirus disease 2019 (COVID-19), which is having an extraordinary impact worldwide [1, 2].

The references in the medical literature regarding the ocular manifestations of this emerging disease are scarce so far and, despite the fact that ocular involvement is not well defined yet, some case reports have highlighted the presence of conjunctivitis [3].

Existing data suggests that conjunctivitis is not a common manifestation associated with COVID-19 [4]. Nonetheless, characterizing conjunctival inflammation in this scenario could be of paramount importance in case it proves to be prevalent as it may be a frequent cause for seeking medical attention in patients possibly infected with SARS-CoV-2 [5].

The vast majority of studies published to date have been carried out in China. To the best of our knowledge, this is the 


\section{Key messages}

- COVID-19 has been shown to have ocular involvement, mainly conjunctivitis.

- Around $12 \%$ of patients affected by COVID-19 can present conjunctivitis symptoms associated with the disease and usually occurs early in the disease.

first study of its kind in Europe. Given the current situation of the SARS-CoV-2 pandemic, describing the clinical characteristics of conjunctivitis associated with the novel coronavirus has relevant implications in the future identification of suspected COVID-19 patients and the differential diagnosis from other forms viral conjunctivitis. The purpose of this study was to evaluate the prevalence of conjunctivitis in hospitalized patients with COVID-19 and to describe its clinical presentation.

\section{Methods}

This cross-sectional study was conducted at the Hospital Clinico San Carlos of Madrid, Spain, a tertiary hospital which attends patients within the Madrid metropolitan area. The study was approved by the Clinical Research Ethics Committee of this institution and was conducted in accordance with the tenets of the Declaration of Helsinki. Informed consent was obtained from all patients.

Hospitalized patients with laboratory-confirmed SARS$\mathrm{CoV}-2$ infection were included. Based on the hospital's protocol, the general admission criteria for patients were as follows: $(1)<50$ years of age without comorbidities with bilateral pneumonia, or unilateral pneumonia with respiratory failure (saturation $<96 \%$ and respiratory rate $>20$ ); or $(2)>$ 50 years of age or patient with comorbidity: with pneumonia, respiratory failure (saturation $<96 \%$ and respiratory rate $>$ 20), or laboratory/clinical severity (arterial blood gas, hemogram, D-dimer, C-reactive protein, procalcitonin, lactate dehydrogenase - LDH, transaminases).

Patients were asked about symptoms of conjunctivitis (current and previous) and they underwent a basic ophthalmological examination at their bedside by two experienced ophthalmologists on a 72-h period. A total of 301 subjects from the COVID admission unit, whose clinical situation allowed us to conduct the aforementioned ophthalmological examination and interview, were systematically explored. To examine the patients, the investigators wore double gloves, a fluid-resistant gown, a full face shield, and both FFP2 and surgical masks.
The inclusion criteria were as follows: over 18 years of age, patient with positive reverse transcriptase-polymerase chain reaction (RT-PCR) test from nasopharyngeal swab for SARS-CoV-2, hospitalized due to COVID-19, and ability to give verbal consent. Those patients admitted to the intensive care unit, unable or unwilling to give verbal consent, and unable to adequately report previous eye symptoms due to general health status were excluded.

The patient's age, sex, the onset of COVID-19 symptoms, chest X-ray, and laboratory tests results were noted. Laboratory work-up included the levels of leukocytes, neutrophils, lymphocytes, C-reactive protein, ferritin, D-dimer, creatinine, and $\mathrm{LDH}$, considering the blood test results that represented the greater severity prior to the date of the ophthalmological examination. Chest X-ray results were analyzed separately, since it is not uncommon to find a discrepancy between the radiological and clinical findings, especially in the early stages of the disease. Additionally, patients were classified according to their clinical severity as mild, moderate, and severe, following the CURB-65 score, physical examination, respiratory assessment (respiratory rate, dyspnea, blood oxygen saturation, ventilation system requirements), or organ failure. The main outcome measure is the overall prevalence of conjunctivitis among inpatients diagnosed with COVID-19.

The overall prevalence results from adding patients who had conjunctivitis at the time of the evaluation and those who reported having conjunctivitis prior to the examination. Prevalence will be presented as a percentage of those with conjunctivitis along with its $95 \%$ confidence interval (CI). The distribution of gender, acute pneumonia, and bilateral pneumonia depending on the outcome status (conjunctivitis or not) will be presented as percentages and the differences addressed through the chi-squared test (of the Fisher's exact test in case the frequencies be low). Differences in prevalence and clinical presentation of conjunctivitis were analyzed by sex. Likewise, the distribution of the quantitative covariates (leucocytes, neutrophils, lymphocytes, C-reactive protein, ferritin, D-dimer, creatinine, and LDH) will be depicted through the median, first, and third quartile, using the Mann-Whitney $U$ test to assess their differences depending on the presence of conjunctivitis and sex. 


\section{Results}

The overall study population included 301 hospitalized patients (601 eyes). Of the 483 patients admitted to the COVID unit at the time of the study, 301 patients met the inclusion and exclusion criteria. A total of 41 patients were admitted to the intensive care unit, 135 patients were unable to adequately report previous eye events due to their clinical situation, cognitive impairment, or confusional state, and 6 patients did not give consent.

Of the 301 subjects included in the study, 180 patients $(59.8 \%)$ were male and the median age was 72 years (IQ $59-82 ; 70$ years in men and 75 years in women, $P=0.13$ ). Overall, 35 patients (11.6\%; 95\% CI: 8.48-15.84) were diagnosed with acute conjunctivitis; of those, 10 (3.3\%; 95\% CI: 1.8-6.1) showed ocular manifestations on the day of the visit whereas 25 (8.3\%; 95\% CI: 5.6-12.1) reported having conjunctivitis in the previous days, seen by the primary care doctor. Upon ophthalmological examination of the 301 patients, other ocular disorders besides conjunctivitis were observed. Specifically, 3 patients presented subconjunctival hemorrhage, 4 patients had a moderate pterygion, and 2 patients had an hordeolum, which were managed with conservative treatment. In addition, a notification system was implemented for all healthcare personnel working at the COVID unit and evaluating the patients daily. Through this system, the on-call ophthalmologist was notified immediately with any new possible case of conjunctivitis until the date of discharge, which contacted the investigators.

Of the 35 cases that presented conjunctivitis, 13 cases suffered it before admission to the hospital, 12 cases in the time interval between admission and our evaluation, and 10 cases presented conjunctivitis at the time of evaluation. The main clinical characteristics found on the latter are shown in Table 1. None of the patients showed conjunctival petechiae, corneal infiltrates nor membranes or pseudomembranes.

The most common reported symptoms among all the 35 patients that presented conjunctivitis during the disease were mucopurulent discharge $(100 \% ; 42.8 \%$ mild, $51.4 \%$ moderate, $5.7 \%$ severe), tearing ( $62.8 \%$ ), and foreign body sensation $(57.1 \%)$. None of the patients of our study reported blurry vision associated.

The median time interval between the onset of COVID-19 symptoms and the appearance of conjunctivitis was 6 days (p25-p75:2-13). There is no statistically significant difference in the time interval from the onset of COVID symptoms to the appearance of conjunctivitis between women and men ( $U$ Mann-Whitney; $P=0.56$ ). According to the patient's self-report, the median duration of ocular symptoms was 3 days (p25-p75: 1-3.5) with a minimum of 1 day and a maximum of 1 week.

Table 2 depicts the distribution of the covariates analyzed depending on the conjunctivitis status, the association between the presence of conjunctivitis, and clinical, laboratory, and radiological data.

$11.6 \%$ of the men and $10 \%$ of the women presented conjunctivitis. Twenty-one $(60 \%)$ patients with conjunctivitis were male and $160(60.1 \%)$ patients without conjunctivitis were male. The association between conjunctivitis and sex did not reach statistical signification $(P=$ $0.98)$.

A total of $262(87.0 \%)$ patients suffered from acute viral pneumonia, which was bilateral in 218 cases $(82.8 \%$ of the pneumonias were bilateral). Twenty-seven (10.3\%) patients with pneumonia and $6(15.3 \%)$ patients without pneumonia presented conjunctivitis. Notwithstanding, Fisher's exact test did not allow us to consider the association between pneumonia and conjunctivitis to be causal $(P=0.40)$. Likewise, the

Table 1 Clinical presentation of conjunctivitis

\begin{tabular}{|c|c|c|c|c|c|c|c|c|c|c|c|c|c|c|}
\hline \multirow[b]{3}{*}{ Conjunctivitis } & \multirow[b]{3}{*}{ Unilateral } & \multicolumn{4}{|c|}{ Overall $(N=35)$} & \multicolumn{4}{|c|}{ Male $(N=21)$} & \multicolumn{4}{|c|}{ Female $(N=14)$} & \multirow{4}{*}{$\begin{array}{l}P \text { value } \\
0.49 *\end{array}$} \\
\hline & & \multirow{3}{*}{$\begin{array}{l}\text { No. } \\
19 \\
16\end{array}$} & \multirow{2}{*}{$\frac{\%}{54.2}$} & \multicolumn{2}{|c|}{$95 \% \mathrm{CI}$} & \multirow{2}{*}{$\begin{array}{c}\text { No. } \\
10\end{array}$} & \multirow{2}{*}{$\frac{\%}{47.7}$} & \multicolumn{2}{|c|}{$95 \% \mathrm{CI}$} & \multirow{3}{*}{$\begin{array}{c}\text { No. } \\
9 \\
5\end{array}$} & \multirow{2}{*}{$\frac{\%}{64.2}$} & \multicolumn{2}{|c|}{$95 \% \mathrm{CI}$} & \\
\hline & & & & 37.1 & 70.5 & & & 26.7 & 69.3 & & & 35.7 & 85.3 & \\
\hline & Bilateral & & 45.7 & 29.5 & 62.9 & 11 & 52.3 & 30.6 & 73.2 & & 35.7 & 14.6 & 64.3 & \\
\hline \multirow[t]{2}{*}{ Conjunctival hyperemia } & Mild & 28 & 80.0 & 62.6 & 90.5 & 17 & 80.9 & 57.1 & 93.1 & 11 & 78.5 & 48.1 & 93.5 & \multirow[t]{2}{*}{$0.99 *$} \\
\hline & Moderate/severe & 7 & 20.0 & 9.4 & 37.4 & 4 & 19.0 & 6.8 & 42.8 & 3 & 21.4 & 6.4 & 51.8 & \\
\hline \multirow[t]{3}{*}{ Mucopurulent discharge } & Mild & 14 & 42.4 & 26.2 & 60.4 & 7 & 35.0 & 16.4 & 59.5 & 7 & 53.8 & 24.8 & 80.4 & \multirow[t]{3}{*}{$0.20 *$} \\
\hline & Moderate & 18 & 54.5 & 36.8 & 71.2 & 13 & 65.0 & 40.4 & 83.5 & 5 & 38.4 & 14.6 & 69.4 & \\
\hline & Severe & 1 & 3.0 & 0.3 & 20.3 & 0 & 0.0 & 0.0 & 0.0 & 1 & 7.6 & 0.7 & 46.8 & \\
\hline \multirow[t]{2}{*}{ Tearing } & Yes & 15 & 42.8 & 27.0 & 60.2 & 9 & 42.8 & 22.6 & 65.8 & 6 & 42.8 & 18.2 & 71.5 & \multirow[t]{2}{*}{$0.99 *$} \\
\hline & No & 20 & 57.1 & 39.7 & 72.9 & 12 & 57.1 & 34.1 & 77.3 & 8 & 57.1 & 28.4 & 81.7 & \\
\hline \multirow[t]{2}{*}{ Foreign body sensation } & Yes & 12 & 34.2 & 20.0 & 52.0 & 8 & 38.1 & 19.0 & 61.6 & 4 & 28.5 & 9.6 & 60.1 & \multirow[t]{2}{*}{$0.72 *$} \\
\hline & No & 23 & 65.7 & 47.9 & 79.9 & 13 & 61.9 & 38.3 & 80.9 & 10 & 71.4 & 39.8 & 90.4 & \\
\hline
\end{tabular}

*Fisher's exact test 
Table 2 Clinical characteristics of patients with and without conjunctivitis

\begin{tabular}{|c|c|c|c|c|c|}
\hline Measure & All $(n=301)$ & $\begin{array}{l}\text { Conjunctivitis } \\
(n=35,11.6 \%)\end{array}$ & $\begin{array}{l}\text { No conjunctivitis } \\
(n=266,88.4 \%)\end{array}$ & $P$ value & \\
\hline Age, median (p25-p75) & $72(59-82)$ & $75(54-85)$ & $71(59-81)$ & 0.38 & $* *$ \\
\hline Male, no. $(\%)$ & $180(59.8 \%)$ & $21(60 \%)$ & $160(60.1 \%)$ & 0.98 & $*$ \\
\hline Female, no. $(\%)$ & $121(40.2 \%)$ & $14(40 \%)$ & $106(39.8 \%)$ & 0.98 & \\
\hline Pneumonia, no. (\%) & $262(87.0 \%)$ & $29(82.8 \%)$ & $233(87.5 \%)$ & 0.42 & $* * *$ \\
\hline Bilateral pneumonia, no. (\%) & $218(82.8 \%)$ & $26(89.6 \%)$ & $192(82.05 \%)$ & 0.43 & $* * *$ \\
\hline Leucocytes, median (p25-p75) & $7(5-9.5)$ & $7.7(4.9-10.4)$ & $7(5-9.4)$ & 0.56 & $* *$ \\
\hline Neutrophils, median (p25-p75) & $5.7(3.7-7.9)$ & $6.4(3.4-8.2)$ & $3.7(5.5-7.9)$ & 0.55 & $* *$ \\
\hline Lymphocytes, median (p25-p75) & $0.6(0.4-1)$ & $0.5(0.3-0.9)$ & $0.6(0.4-1)$ & 0.32 & $* *$ \\
\hline CRP, median (p25-p75) & $7.22(2.2-15.2)$ & $6.75(1.8-14.9)$ & $7.25(2.3-15.7)$ & 0.68 & $* *$ \\
\hline Ferritin, median (p25-p75) & $589.6(287.3-1125.1)$ & $548.3(224.6-948.8)$ & $591.8(29.8-1150.8)$ & 0.14 & $* *$ \\
\hline D-dimer, median (p25-p75) & $1075(606-2146)$ & $1126(577-2254)$ & $1073.5(622-2146)$ & 0.89 & $* *$ \\
\hline Creatine, median (p25-p75) & $0.81(0.62-1.13)$ & $0.88(0.66-1.37)$ & $0.805(0.6-1.1)$ & 0.29 & $* *$ \\
\hline LDH, median (p25-p75) & $633(482-8269$ & $613(463-886)$ & $635.5(482-825)$ & 0.94 & $* *$ \\
\hline
\end{tabular}

*Chi squared

**U Mann-Whitney

****Fisher's exact test

$L D H$, lactate dehydrogenase; $C R P, \mathrm{C}$-reactive protein

association between the laterality of the pneumonic process was not significantly associated with the presence of conjunctivitis $(P=0.18)$.
Table 3 illustrates the laboratory, radiological, and clinical results in patients with and without conjunctivitis stratified by sex.

Table 3 Laboratory, radiological, and clinical results in patients with and without conjunctivitis stratified by sex

\begin{tabular}{|c|c|c|c|c|c|c|c|c|c|c|c|c|c|c|}
\hline \multirow[b]{3}{*}{ Variables } & \multicolumn{7}{|c|}{ Conjunctivitis } & \multicolumn{7}{|c|}{ No conjunctivitis } \\
\hline & \multicolumn{3}{|l|}{ Male } & \multicolumn{3}{|c|}{ Female } & \multirow[t]{2}{*}{$P$} & \multicolumn{3}{|l|}{ Male } & \multicolumn{3}{|c|}{ Female } & \multirow[t]{2}{*}{$P$ value } \\
\hline & p50 & p25 & p75 & p50 & p25 & p75 & & p50 & p25 & p75 & p50 & p25 & p75 & \\
\hline Leucocytes & 9 & 6.1 & 10.5 & 6 & 4 & 9 & $0.03 *$ & 7.1 & 5 & 9.45 & 6.6 & 4.9 & 9.1 & $0.58^{*}$ \\
\hline Neutrophils & 7.4 & 5.2 & 9.1 & 4 & 3 & 8 & $0.04 *$ & 5.9 & 3.7 & 8 & 5.3 & 3.4 & 7.9 & $0.20 *$ \\
\hline Lymphocytes & 0.5 & 0.3 & 0.8 & 1 & 0 & 1 & $0.68^{*}$ & 0.6 & 0.4 & 0.8 & 0.7 & 0.4 & 1.1 & $0.01 *$ \\
\hline PCR & 9.64 & 2.32 & 14.9 & 4 & 2 & 12 & $0.34^{*}$ & 9.1 & 3.4 & 17.25 & 5.32 & 1.13 & 11.2 & $0.001 *$ \\
\hline Ferritin & 654.2 & 313.9 & 1035.1 & 268 & 103 & 734 & $0.04 *$ & 859.6 & 389.5 & 1353.6 & 374.5 & 201.1 & 724.4 & $<0.001 *$ \\
\hline D-dimer & 1700 & 618 & 4513 & 675 & 380 & 1127 & $0.01^{*}$ & 1088.5 & 663.5 & 2117.5 & 1008 & 512 & 2232 & $0.29 *$ \\
\hline Creatinine & 1.07 & 0.82 & 2 & 1 & 1 & 1 & $0.03 *$ & 0.8 & 0.6 & 1.19 & 0.69 & 0.54 & 0.93 & $<0.001 *$ \\
\hline LDH & 622 & 485 & 886 & 591 & 408 & 699 & $0.59^{*}$ & 635.5 & 491.5 & 794.5 & 640 & 466 & 871 & $0.62 *$ \\
\hline Variable & \multicolumn{3}{|c|}{ No. $(\%)$} & \multicolumn{4}{|c|}{ No. $(\%)$} & \multicolumn{3}{|c|}{ No. $(\%)$} & \multicolumn{3}{|c|}{ No. (\%) } & \\
\hline Pneumonia & \multicolumn{3}{|c|}{$17(80.9 \%)$} & \multicolumn{3}{|c|}{$12(85.7 \%)$} & $0.99 * * *$ & \multicolumn{3}{|c|}{$138(86.2 \%)$} & \multicolumn{3}{|c|}{$95(89.6 \%)$} & $0.41 * *$ \\
\hline Pneumonia bilat & \multicolumn{3}{|c|}{$16(94.1 \%)$} & \multicolumn{3}{|c|}{$10(83.3 \%)$} & $0.55^{* * *}$ & \multicolumn{3}{|c|}{$114(82.0 \%)$} & \multicolumn{3}{|c|}{$78(82.1 \%)$} & $0.98 * *$ \\
\hline \multicolumn{15}{|l|}{ Severity } \\
\hline Mild & \multirow{2}{*}{\multicolumn{3}{|c|}{$\begin{array}{l}1(4.7 \%) \\
12(57.1 \%)\end{array}$}} & \multirow{2}{*}{\multicolumn{3}{|c|}{$\begin{array}{l}9(64.2 \%) \\
3(21.4 \%)\end{array}$}} & $0.001 * * *$ & \multicolumn{3}{|c|}{$68(42.0 \%)$} & \multicolumn{3}{|c|}{$48(45.2 \%)$} & $0.17 * *$ \\
\hline Moderate & & & & & & & & $53(33.1$ & & & & & & \\
\hline Severe & \multicolumn{3}{|c|}{$8(38.1 \%)$} & \multicolumn{4}{|c|}{$2(14.2 \%)$} & \multicolumn{3}{|c|}{$39(24.3 \%)$} & \multicolumn{3}{|c|}{$16(15.1 \%)$} & \\
\hline
\end{tabular}

*U Mann-Whitney

$* * Z$-test

****Fisher's exact test 
Table 4 Differences in clinical severity by gender among all patients and those with conjunctivitis

\begin{tabular}{|c|c|c|c|}
\hline \multicolumn{4}{|c|}{ All patients $(N=301)$} \\
\hline Variable & Male & Female & $P$ value \\
\hline \multicolumn{4}{|l|}{ Severity: } \\
\hline 1 & $69(38.1 \%)$ & $57(47.5 \%)$ & \multirow[t]{3}{*}{$0.06^{*}$} \\
\hline 2 & $65(35.9 \%)$ & $45(37.5 \%)$ & \\
\hline 3 & $47(25.9 \%)$ & $18(15 \%)$ & \\
\hline \multicolumn{4}{|c|}{ Conjunctivitis $(N=35)$} \\
\hline & Male & Female & $P$ \\
\hline \multicolumn{4}{|l|}{ Severity: } \\
\hline 1 & $1(4.7 \%)$ & $9(64.2 \%)$ & \multirow[t]{3}{*}{$0.001 * *$} \\
\hline 2 & $12(57.1 \%)$ & $3(21.4 \%)$ & \\
\hline 3 & $8(38.1 \%)$ & $2(14.2 \%)$ & \\
\hline
\end{tabular}

*Chi-squared test

**Fisher's exact test

Among the 301 patients, $41.8 \%$ were classified as mild, $36.5 \%$ cases classified as moderate, and $21.5 \%$ classified as severe disease. The biochemical profiles in men and women also showed differences between them. According to the chisquared test, there is not a relationship between the COVID19 severity score and the presence of conjunctivitis $(P=0.17)$. However, in the analysis of conjunctivitis patients by clinical severity, there were statistically significant differences by sex (Table 4). Conjunctivitis was more frequent in males with moderate clinical severity and in women classified as clinically mild.

\section{Discussion}

Coronavirus disease 2019 (COVID-19) has shown several clinical manifestations at respiratory, gastrointestinal, and neurological levels, among others [2,4]. Although the most frequent symptoms include respiratory symptoms, such as fever, cough, and dyspnea, the presence of conjunctivitis has also been reported [3].

The frequency of conjunctivitis in patients with COVID-19 has not been fully quantified to date, reporting very different data regarding its prevalence and incidence. A study analyzing a sample of 1099 patients hospitalized for COVID-19 disease in China found a prevalence of conjunctivitis symptoms of only $0.8 \%$ and other small series have reported a prevalence around 3\% [4, 6, 7]. However, Wu P. et al. [8] found that as high as $31.6 \%$ (95\% CI, 17.5-48.7) of hospitalized COVID19 patients presented ocular signs and symptoms compatible with conjunctivitis. Our results show an $11.6 \%$ prevalence of conjunctivitis among hospitalized patients with COVID-19, differing from previous results.

Conjunctivitis as a presenting manifestation of coronavirus and the relationship between conjunctivitis and the development of serious pulmonary disease are important questions for ophthalmologists worldwide. Wu et al. [8] described in a series of 38 patients that patients with conjunctivitis were more likely to have higher white blood cell and neutrophil counts and higher levels of procalcitonin, Creactive protein, and lactate dehydrogenase than patients without ocular symptoms, suggesting that ocular abnormalities occurred more frequently in patients with more severe COVID-19. However, this article did not compare the differences between women and men, and included critical patients, who were not included in our sample. We were unable to objectify any relationship between the presence of conjunctivitis and clinical, radiological, or laboratory severity in our sample of 301 cases. Furthermore, it is interesting to highlight that out of a hospitalized married couple who slept in the same room at home and had equal clinical severity, only the woman presented with conjunctivitis. This suggests that perhaps the appearance of conjunctivitis could depend on the host's characteristics or the inoculation mechanism.

Studies suggest that there are many differences between men and women in the immune response to SARS-CoV-19, affecting more men than women $[9,10]$. Despite our study showed no difference in the clinical presentation of conjunctivitis in male and female, we found that conjunctivitis was more frequent in males with moderate COVID-19 and women with mild disease. We believe this is related to males having a more severe biochemical COVID-19 profile than females.

The clinical characteristics found in conjunctivitis associated with SARS-CoV-2 infection showed common aspects with other viral conjunctivitis, such as follicular reaction, and conjunctival hyperemia and discharge. However, we found distinctive clinical findings among our patients that could guide defining conjunctivitis in COVID-19 patients.

The differential diagnosis of SARS-CoV-2 conjunctivitis includes other viral conjunctivitis such as adenoviral conjunctivitis, based on our findings (Table 5). 54.29\% of conjunctivitis were completely unilateral, unlike adenoviral conjunctivitis where there is a greater tendency to bilateralization [11]. However, bilateral conjunctivitis in COVID-19 has also been described [3].

The degree of conjunctival hyperemia was mild or very mild and the presence of follicular reaction has also been reported by other authors [3]. It was striking to find the absence of petechiae and subconjunctival hemorrhages in our sample, despite the fact that different articles reported the vascular and thrombotic complications associated with the virus $[12,13]$. Also, we did not find any associated complications such as corneal infiltrates and membranes or pseudomembranes, which have not been reported in the literature so far.

The natural history of the disease seems to be a rapid selflimited conjunctivitis that improves without specific treatment. On the other hand, adenoviral conjunctivitis tends to 
Table 5 Differential diagnosis between conjunctivitis in COVID-19 patients and adenoviral conjunctivitis

\begin{tabular}{lll}
\hline & Conjunctivitis in COVID-19 & Adenoviral conjunctivitis \\
\hline Onset & Abrupt & Abrupt \\
Unilateral/bilateral & Unilateral ++ & $\begin{array}{c}\text { Unilateral or bilateral } \\
\text { (often sequentially bilateral) }\end{array}$ \\
Conjunctival injection & Bilateral + & Varies in severity \\
Follicular reaction & Mild or very mild & + \\
Chemosis & + & \pm \\
Eyelid swelling and erythema & \pm & \pm \\
Conjunctival petechiae/subconjunctival hemorrhage & - & \pm \\
Discharge & + & ++ \\
Epithelial punctate keratitis & - & \pm \\
Corneal infiltrates & - & \pm \\
Membrane/pseudomembrane formation & - & \pm \\
Concurrent upper respiratory tract infection & \pm & \pm \\
Symptoms & Very mild-mild & Mild-severe \\
Natural history & Self-limited within 2-4 days & Self-limited within 5-14 days \\
& & (could get worse during the first days) \\
Potential sequelae & Not Known & Severe cases: conjunctival scarring, symblepharon, \\
& & subepithelial corneal infiltrates, decreased vision \\
\hline
\end{tabular}

worsen during the first days and could last more than 14 days. The onset of conjunctivitis signs and symptoms with respect to the onset of respiratory symptoms was variable (median of 3 days). Previous reports suggest that SARS-CoV-2 can cause conjunctivitis, either as an early sign of infection or during hospitalization for severe COVID-19 [8, 14].

The fact that none of the patients reported associated blurred vision nor shown relevant epithelial corneal keratitis agrees with the case series of Wu et al. [8] However, there is a recent case report that described keratoconjunctivitis as the initial medical presentation of a patient with COVID-19 [15].

Limitations of this study include the absence of detailed ocular examinations (dilated fundus exam) to exclude intraocular disease owing to the logistical challenges of managing these patients at this critical healthcare resource situation. Since this a cross-sectional study and as such, patients that had conjunctivitis prior to admission were not evaluated by the investigators, but they were evaluated by primary care physicians.

The demonstration of the direct association between conjunctivitis and SARS-CoV-2 infection in the absence of diagnostic confirmation with real-time polymerase chain reaction (RT-PCR) of tears and conjunctival secretions is difficult to prove. However, based on previous results, the extremely low positive rate of SARS-CoV-2 RNA test by RT-PCR in tears and conjunctival secretions from patients with laboratoryconfirmed SARS-CoV-2 implies that negative test results could be false negative, not excluding the presence of the virus [8, 16]. For this reason and due to the limited resources and restrictive measures of access to patients with COVID-19, RT-PCR from tears and conjunctival specimen was not tested. Based on our findings, we estimate that the actual prevalence could be underestimated, partly because many mild or very mild cases may have gone unnoticed by both healthcare personnel and the patients themselves. The exclusion of patients with cognitive impairment or confusional syndrome suggests there could also imply a shift in the actual prevalence. Since this is a tertiary hospital in downtown Madrid that covers a health area with an aging population, a high number of patients had to be excluded due to cognitive impairment, confusional state, and critical conditions, in order to obtain more reliable data. These excluded patients showed no difference in clinical characteristics compared with the included sample.

This is the first study that describes the clinical characteristics of conjunctivitis in a large sample of patients with COVID19. A recent study carried out in China reported that the prevalence of conjunctival congestion in COVID-19 patients was $5 \%$ [17]. However, out of the 535 patients included, only 343 patients $(64.1 \%)$ had laboratory-confirmed SARS-CoV-2 infection from nasopharyngeal swabs. Our study includes a total of 301 patients, all of them with the laboratory-confirmed diagnosis. Moreover, the above-mentioned article is a retrospective study where the patients were not evaluated by an ophthalmologist, and the patient's data were obtained from patients' electronic medical records and an electronic questionnaire completed by patients on a smartphone. Furthermore, data about ocular manifestations were obtained by ophthalmologists via telephone, so results are determined by patient subjectivity and ophthalmologist's interpretation. This makes our study the most comprehensive and extensive of its category. 
COVID-19 has spread rapidly since it was first identified in Wuhan and has been shown to have ocular involvement, mainly conjunctivitis. We found a prevalence of conjunctivitis in our sample of $11.6 \%$, which allows us to infer that approximately 1 in 10 patients affected by COVID-19 can present conjunctivitis symptoms associated with the disease.

Our observations can help ophthalmologists and other physicians to identify possible COVID-19 patients presenting with red eye or discharge as main complain for seeking care, especially in women who could present conjunctivitis earlier in the disease. However, at this point in the COVID-19 pandemic, it is reasonable that practically any patient seen by a medical practitioner is considered suspected of SARS-CoV-2 infection, regardless of presenting signs or symptoms of conjunctivitis.

A better understanding of the ocular manifestations of the virus will assist in early identification of SARS-CoV-2infected cases, prioritizing diagnostic testing in patients with clinical findings compatible with conjunctivitis associated with COVID-19.

Data availability Data sharing is not applicable to this article as no datasets were generated or analyzed during the current study.

\section{Compliance with ethical standards}

This article is based on previously conducted studies and does not contain any studies with human participants or animals performed by any of the authors. All named authors meet the International Committee of Medical Journal Editors (ICMJE) criteria for authorship for this article, take responsibility for the integrity of the work as a whole, and have given their approval for this version to be published.

Conflict of interest The authors declare that they have no conflict of interest.

\section{References}

1. Huang Y, Tu M, Wang S et al (2020) Clinical characteristics of laboratory confirmed positive cases of SARS-CoV-2 infection in Wuhan, China: a retrospective single center analysis. Travel Med Infect Dis:101606. https://doi.org/10.1016/j.tmaid.2020.101606

2. Huang C, Wang Y, Li X et al (2020) Clinical features of patients infected with 2019 novel coronavirus in Wuhan, China. Lancet 395(10223):497-506. https://doi.org/10.1016/S0140-6736(20) 30183-5

3. Chen L, Liu M, Zhang Z et al (2020) Ocular manifestations of a hospitalised patient with confirmed 2019 novel coronavirus disease. Br J Ophthalmol:1-4. https://doi.org/10.1136/ bjophthalmol-2020-316304

4. Guan W, Ni Z, Hu Y et al (2020) Clinical characteristics of coronavirus disease 2019 in China. N Engl J Med:1-13. https://doi.org/ 10.1056/nejmoa2002032

5. Ramirez DA, Porco TC, Lietman TM, Keenan JD (2017) Epidemiology of conjunctivitis in US emergency departments. JAMA Ophthalmol 135(10):1119-1121. https://doi.org/10.1001/ jamaophthalmol.2017.3319

6. Xia J, Tong J, Liu M, Shen Y, Guo D (2020) Evaluation of coronavirus in tears and conjunctival secretions of patients with SARSCoV-2 infection. J Med Virol:1-6. https://doi.org/10.1002/jmv. 25725

7. Zhang X, Chen X, Chen L et al (2020) The evidence of SARSCoV-2 infection on ocular surface. Ocul Surf. https://doi.org/10. 1016/j.jtos.2020.03.010

8. Wu P, Duan F, Luo C et al (2020) Characteristics of ocular findings of patients with coronavirus disease 2019 (COVID-19) in Hubei Province, China. JAMA Ophthalmol:1-8. https://doi.org/10.1001/ jamaophthalmol.2020.1291

9. Conti P, Younes A (2020) Coronavirus COV-19/SARS-CoV-2 affects women less than men: clinical response to viral infection. $\mathrm{J}$ Biol Regul Homeost Agents 34(2). https://doi.org/10.23812/ Editorial-Conti-3

10. Yi Y, Lagniton PNP, Ye S, Li E, Xu R-H (2020) COVID-19: what has been learned and to be learned about the novel coronavirus disease. Int J Biol Sci 16(10):1753-1766. https://doi.org/10.7150/ ijbs. 45134

11. Yeu E, Hauswirth S (2020) A review of the differential diagnosis of acute infectious conjunctivitis: implications for treatment and management. Clin Ophthalmol 14:805-813. https://doi.org/10.2147/ OPTH.S236571

12. Giannis D, Ziogas IA, Gianni P (2020) Coagulation disorders in coronavirus infected patients: COVID-19, SARS-CoV-1, MERS$\mathrm{CoV}$ and lessons from the past. J Clin Virol 127:104362. https://doi. org/10.1016/j.jcv.2020.104362

13. Xiong M, Liang X, Wei Y-D (2020) Changes in blood coagulation in patients with severe coronavirus disease 2019 (COVID-19): a meta-analysis. Br J Haematol. https://doi.org/10.1111/bjh.16725

14. Hu K, Patel J, Patel BC (2020) Ophthalmic manifestations of coronavirus (COVID-19). StatPearls 9:1-9 http://www.ncbi.nlm.nih. gov/pubmed $/ 32310553$

15. Cheema M, Aghazadeh H, Nazarali S et al (2020) Keratoconjunctivitis as the initial medical presentation of the novel coronavirus disease 2019 (COVID-19): a case report. Can J Ophthalmol. https://doi.org/10.1016/j.jcjo.2020.03.003

16. Zhou Y, Duan C, Zeng Y et al (2020) Ocular findings and proportion with conjunctival SARS-COV-2 in COVID-19 patients. Ophthalmology. https://doi.org/10.1016/j.ophtha.2020.04.028

17. Chen L, Deng C, Chen X et al (2020) Ocular manifestations and clinical characteristics of 535 cases of COVID-19 in Wuhan, China: a cross-sectional study. Acta Ophthalmol. https://doi.org/10.1111/ aos. 14472

Publisher's note Springer Nature remains neutral with regard to jurisdictional claims in published maps and institutional affiliations. 